H. Simmer in Niklasdorf a. d. Mur in Steiermark, der Herausgeber der ,Kryptogamen des Kreuzeckgebietes" (bisher 4 Centurien), beabsichtigt ein nenes Exsiccatenwerk: „Kryptogamen des obersteierischen Eragebirges"6 herauszugeben. Preis pro Centurie $K$ 20.-.

\title{
Personal-Nachrichten.
}

\section{Ernannt wurden:}

Berlin.

Dr. Ernst Gilg zum Custos am kgl. Botanischen Museum zu

Dr. Hermann Harms zum wissenschaftlichen Beamten für Botanik an der kgl. Akademie der Wissenschaften zu Berlin.

Dr. Bohumil Nèmec zum Vorstand des neven pflanzenphysiologischen Instituts an der k. k. böhmischen Universität in Prag.

Prof. Dr. Wladimir Iwan Palladin (Warschan) zum ord. Professor der Anatomie und Physiologie der Pflanzen an der kais. Universität in St. Petersburg.

Dr. Oskar Uhlworm (Herausgeber des „Botan. Centralblatt") zum Bibliothekar an der kgl. Bibliothek zu Berlin und zum Vorstand des Deutschen Bureaus für internationale Bibliographie.

Gestorben sind:

Der Sinologe Dr. Emil Bretschneider in St. Petersburg.

Der Algologe Dr. Antonio Pi c c o ne, Professor am Liceo Cristoforo Colombo in Genua am 21. Mai im 58. Lebensjabre.

\section{Druckfehler-Berichtigung.}

In Nr. 7 des laufenden Jahrganges dieser Zeitschrift auf S. 269, Zeile 3 von oben ist zu setzen: „Kältemischung" statt „Kalkmischung“.

Inhalt der August-Nummer: Dr. A. Zahlbruekner, Vorabeiten za einer Flechtenflora Dalmatiens. S. 273. - Victor Schiffner, Neue Untersuchungen ther Calycularia crispula und Calycularia birmensis. S. 285. - E. H a ckel. Neue Gräser. S. 290. - Dr. A u gust v. Hayek, Beiträge zur Flora von Steiermark. (Forts.) S. 295. - Mari e! Soltoković, Die perennen Arten der Gattung Gentiana aus der Section Cyclostigma. (Schluss.) S. 304. Literatur-Uebersicht. S. 312. - Akademien, Botanische Gesellschaften, Vereine, Congresse etc. S. 317. - Botanische Sammlungen, Museen, Institute etc. S. 318. - Personal-Nachrichten. S. 319 .

Redacteur: Prof. Dr. R. ฬ. Wettstein, Wien, 3/3, Rennweg 14.

Verantwortlicher Redacteur: J. Dörfler, Wien, III., Barichgasse 36. Verlag von Carl Gerold's Sohn in Wien.

Die "Oesterreiohische botanische Zeitsohrift" erscheint am Ersten eines jeden Monates nnd kostet ganzjöhrig 16 Mark.

Zu herabgesetzten Preisen sind noch folgende Jahrgănge der Zeitschrift zu haben:

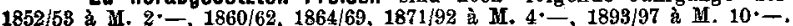

Exemplare, die frei durch die Post expedirt werden sollen, sind mittelst Postanweisnng direct bei der Administration in Wien, I., Barbaragasse 2 (Firma Carl Gerold's Sohn), zu pränumeriren. Einzelne Nummern, soweit noch vorräthig, à 2 Mark.

Ankündigungen werden mit 30 Pfennigen für die durchlaufende Petitzeile berechnet. 